\title{
La Activación Conductual en la práctica: técnicas, organización de la intervención, dificultades y variantes
}

\author{
Jorge Barraca \\ Universidad Camilo José Cela
}

\section{RESUMEN}

Este artículo presenta información centrada en la aplicación de la Activación Conductual y las dificultades que pueden aparecer durante su puesta en práctica. Tras recodar brevemente su punto de partida como terapia independiente y explicar su modelo teórico que da cuenta del origen y del mantenimiento de los estados depresivos, se presenta una información más detallada sobre las técnicas de intervención, así como de la secuencia que se aconseja para su aplicación en el contexto clínico. A partir de una numeración, se describen los pasos que parecen más recomendables según una integración de los principales protocolos de la terapia. Así mismo, se presentan algunas pistas para el manejo de las situaciones difíciles más características de la aplicación de la Activación Conductual. Finalmente, se repasan algunos de los ámbitos hacia los que se ha expandido esta intervención, más allá de la depresión unipolar. Una conclusiones sobre su papel e importancia como herramienta para la lucha contra la depresión desde el plano de la terapia psicológica cierran este trabajo.

Palabras clave: Activación Conductual; depresión; aplicación clínica; dificultades en la aplicación; variaciones en la aplicación.

\section{ABSTRACT}

This article presents information focused on the application of Behavioral Activation and the difficulties that may arise during its implementation. After briefly remind its origin as an independent therapy and explain their theoretical model that accounts for the origin and maintenance of depressive moods, more detailed information on intervention techniques and the recommended sequence for its application in the clinical context is submitted. Through a list that integrates the core protocols of therapy, the more appropriate steps are put forward. Also, some clues for handling the most difficult and common situations of the application of Behavioral Activation are presented. Finally, some of the areas to which this intervention has expanded beyond unipolar depression are reviewing. Some conclusions about its role and importance as a tool for fighting depression from the perspective of psychological therapy ends this work.

Keywords: Behavioral Activation; depression; clinical application; application difficulties; application variants. 
La Activación Conductual (AC, en adelante) puede definirse como un tratamiento estructurado, parsimonioso en su aplicación, teóricamente fundamentado y ya bien establecido como terapia con fuerte apoyo empírico para el tratamiento de la depresión. A día de hoy representa para los terapeutas la alternativa más contrastada y ventajosa frente a la intervención cognitiva propuesta desde el modelo de Beck (Beck, Rusch, Shaw, \& Emery, 1979), estándar de calidad hasta el momento presente. Por supuesto, no es la única terapia eficaz para la depresión: la misma propuesta cognitiva de Beck ha reunido incontestables evidencias de utilidad (DeRubeis \& Crits-Christoph, 1998), como también existen pruebas sólidas para el Análisis de Sistemas Cognitivo-Conductual de Psicoterapia, La Psicoterapia Interpersonal, la Terapia de Solución de Problemas o la Terapia de Autogestión/Autocontrol (APA Division 12, 2015). No obstante, si se tiene en cuenta la duración de la intervención y el número de estrategias empleadas, la severidad de los cuadros en que ha tenido éxito, el rango de cambio en las puntuaciones de los cuestionarios con propiedades psicométricas más contrastadas, la ausencia de recaídas tras dos años de tratamiento, el abandono de fármacos antidepresivos y el rigor metodológico de los ensayos clínicos aleatorizados presentados como evidencia, la AC se corona como la opción terapéutica que aúna más ventajas para el clínico y el cliente.

La AC cuenta ya además con un conjunto de publicaciones en cantidad y calidad suficiente para que su divulgación, tanto en español como en inglés, no represente un obstácu- lo; si bien es cierto que no existen tantas posibilidades para formarse en la práctica a través de talleres y cursos especializados fuera de los Estados Unidos, así como contar con la supervisión profesional de un terapeuta experto en $A C$ o con los materiales más actualizados (cuestionarios, registros, aplicaciones móviles, etc.) para su implementación en la terapia.

A lo largo de las páginas siguientes se ofrece una presentación actualizada de la AC en la que se menciona su origen y se exponen los aspectos fundamentales de sus presupuestos teóricos y su modelo explicativo de la depresión, pero sobre todo se recorren sus técnicas de intervención, se muestra la secuencia de su puesta en funcionamiento y se comentan los problemas más habituales al llevarla a la práctica. Además, se mencionarán las publicaciones más recientes que acogen algunas variantes en su aplicación cuando se extiende su foco de interés más allá de la depresión unipolar.

\section{El origen de la AC para la depresión}

Aunque una parte importante de la base teórica de la AC pueden remontarse a las propuestas de Ferster (1973) y Lewinsohn (1974), en realidad como terapia autónoma su origen está en los trabajos Neil Jacobson sobre el desmantelamiento de los componentes eficaces de la terapia cognitiva de Beck (Gortner, GoIlan, Dobson, \& Jacobson, 1998; Jacobson et al., 1996). En estos estudios se reveló que la inclusión de las estrategias puramente cognitivas no suponía una mejora significativa para los casos en los que ya se habían incorporado los componentes conductuales. Por tanto, el desarrollo de una terapia que se conformase exclu- 
sivamente a partir técnicas conductuales (en particular la programación jerarquizada de actividades) resultaría más parsimoniosa e igual de eficaz que todo el paquete de tratamiento planteado por Beck (activación conductual + evaluación y reconocimiento de pensamientos automáticos + reestructuración cognitiva).

En coherencia con estos resultados, solo faltaba desarrollar un protocolo de tratamiento para su uso en ensayos clínicos que añadiese algunos aspectos novedosos respecto a las aportaciones de Ferster, Lewinsohn o del mismo Beck. Estos aspectos pueden sintetizarse en: (1) una elaboración teórica mejor perfilada, aunque sencilla, para explicar tanto el origen como la perpetuación del estado depresivo (que se expondría a los pacientes como modelo explicativo); (2) un nuevo enfoque sobre aspectos motivacionales para comprometer al cliente en la intervención (la necesidad de 'hacer para luego encontrarse bien', y no al revés); (3) un protocolo de tratamiento bien desarrollado, organizado jerárquicamente y formalizado a partir de las técnicas de activación conductual más contrastadas en los ensayos clínicos; (4) una perspectiva y un manejo genuinamente conductual de los pensamientos, en que la rumia depresiva se aborda desde una perspectiva funcional; (5) el desarrollo de algunos instrumentos de evaluación originales para valorar el curso de la terapia; y (6) la inclusión del concepto de valores - una temática propia de las terapias de tercera generaciónpara facilitar la elección de las conductas a activar (Barraca, 2009). Así, tras emplear un manual propio en la investigación, los autores de la terapia publicaron sendos textos (Jacobson,
Martell, \& Dimidjian, 2001; Martell, Addis, \& Jacobson, 2001) donde se explicaba ya para su divulgación entre profesionales cómo llevar a cabo esta terapia, que a partir de ese momento denominaron "Activación Conductual".

Debe también aclararse que, prácticamente en las mismas fechas, Lejuez, Hopko y Hopko (2001), Lejuez, Hopko, LePage, Hopko y McNeil (2001) presentaron un modelo de tratamiento de AC más breve y más protocolizado, (y con un cuerpo de evidencia empírica menor en ese momento) que denominaran "Terapia de Activación Conductual para la Depresión" (o “Terapia Breve de Activación Conductual para la Depresión"). Si bien ambos modelos tienen sus diferencias a la hora de llevar a la práctica la terapia y enfatizan diversos aspectos (Barraca, 2009; Hopko, Lejuez, Ruggiero, \& Eifert, 2003), respecto al punto básico -la salida de la depresión se logra a través de la estimulación y recuperación de actividades valiosas para el sujeto- pueden considerarse parejos y fruto de una idea esencial común.

\section{El modelo teórico de la AC}

La AC no parte de un modelo psicopatológico al uso, antes bien aboga por deslindarse de enfoques explicativos derivados más directamente de la investigación clínica convencional y recuperar, en cambio, conceptos filosóficos o históricos que despatologizan (o desmedicalizan) los estados de ánimo bajos (Barraca \& Pérez-Álvarez, 2015; Pérez-Álvarez, 2007). La depresión, que ya no se vería como un estado morboso sino como una respuesta normal o esperable ante un determinado contexto vital, se explicaría a partir de las circuns- 
tancias presentes en ese momento y se afianzaría fundamentalmente como consecuencia de las mismas respuestas o reacciones del individuo a ese entorno. En consecuencia, como menciona Martell (2015), la AC sostiene que es el contexto y no factores internos (como determinadas cogniciones o desequilibrios de los neutrotransmisores) lo que aporta una explicación de la depresión más eficiente y representa un ámbito de intervención más eficaz. La AC buscaría ayudar a las personas a comprender las fuentes ambientales de su depresión y localizar aquellas conductas que están manteniendo o empeorando la depresión.

De acuerdo con el planteamiento del modelo expuesto más extensamente en Jacobson et al. (2001), Martell et al. (2001) y Martell, Dimidjian y Herman-Dunn, 2010) para la AC el inicio de la depresión es consecuencia de un suceso (o conjunto de sucesos) que, de forma súbita o progresiva, aparta de la vida del sujeto reforzadores capitales. Aunque se reconoce el posible concurso de factores genéticos, biológicos u otros en la génesis del cuadro, el modelo enfatiza el papel de las pérdidas vitales como elicitadores, al menos, de algunas depresiones. La perpetuación de este estado se debería al mantenimiento de unas conductas que, si bien suponen un alivio a corto plazo, impiden romper la dinámica que encadena al sujeto a una vida pobre en reforzamiento positivo. Es en este sentido en el que se afirma que las "conductas depresivas" están bajo un paradigma de reforzamiento negativo. En conclusión y de forma elemental: la depresión se mantendría porque el cliente evita fuentes potenciales y naturales de reforzamiento (que serían antidepresivas) debido a que el contacto con estas es demasiado desafiante, amenazante, doloroso o incómodo en ese momento de su vida. Esta evitación lleva a un alivio inmediato, pero mantiene la depresión a la larga, tanto porque no se experimentan gratificaciones enjundiosas, cuanto porque los estresores vitales empeoran con el paso del tiempo. Por eso, la clave de la intervención radica en ayudar a los clientes a reexperimentar el contacto con las fuentes de recompensa de su vida (por medio de la activación de determinadas acciones que harían recuperar el contacto con los reforzadores naturales y la extinción de las acciones que son evitaciones) y volver a vivir una vida que merezca la pena (Barraca \& Pérez-Álvarez, 2015). Como puede comprobarse, la depresión es vista así como un proceso contextual en el que no se cree que jueguen un papel variables cognitivas previas, modificaciones cerebrales o cambios químicos. Aunque es posible que el malestar tenga sus correlatos en forma de determinados pensamientos o en un déficit de la serotonina (por poner un ejemplo), estos no serían nunca factores causales, sino, si acaso, consecuentes, y por tanto el tratamiento que procurase su cambio directo resultaría inútil a la larga.

\section{La práctica de la AC:}

\section{técnicas y secuencia de intervención}

Las técnicas que se emplean en la $A C$ son conocidas en la tradición de la modificación de conducta. Precisamente, una de las bondades de esta terapia radica en que se ha decantado por unos pocos métodos de contrastada eficacia y de sencilla aplicación. Estos métodos 
son: (1) la programación y estructuración de actividades; (2) la solución de problemas; (3) el reforzamiento positivo directo; (4) el desvanecimiento; (5) el entrenamiento en habilidades sociales; y (6) los métodos para facilitar un contacto directo con la experiencia.

Estas técnicas se diferencian respecto a su peso en la intervención: la programación de actividades (jerarquizadas, si es necesario), que se utiliza para recuperar el contacto con los reforzadores naturales, es el procedimiento fundamental que se incluye en todos los casos y conforma la columna vertebral de la intervención. En segundo lugar, aunque muchas veces de forma complementaria, también se incorpora la extinción de las conductas de evitación, siempre que estas no desaparezcan con la misma programación, y que se guía con un procedimiento simplificado de solución de problemas. En tercer lugar -de forma ya menos frecuente- se recurre en algunos casos al reforzamiento positivo directo (autoreforzamiento y heteroreforzamiento, que se puede formalizar a través de contratos conductuales) y que se emplea para motivar al sujeto hasta que los reforzadores naturales lleguen a ser los que controlen la conducta. Si algún déficit conductual impide o hace muy difícil obtener los reforzadores naturales, se entrenan secuencias básicas de habilidades sociales, de ensayos mentales o se usa el desvanecimiento (de las ayudas y facilitaciones del terapeuta o de familiares). Por último, si el terapeuta observa que el pensamiento rumiante impide un buen contacto con los reforzadores, se recurre a la "atención a la experiencia" (que se sirve del mindfulness $y / o$ de otros procedimientos) para que al sujeto le resulte más fácil apreciar el impacto de esos reforzadores en su vida (Barraca \& Pérez-Álvarez, 2015).

Conocer la secuencia de intervención que sigue la AC es tan importante como comprender y dominar las técnicas que acaban de mencionarse. Saber cómo se pasa de la fase de evaluación a la de intervención, o de qué manera se organiza y estructura la activación resulta fundamental. Hay que recordar que la $A C$ se ha definido como una intervención estructurada, aunque no estrictamente protocolizada. Esto es así porque se presenta como un enfoque de naturaleza altamente idiográfica. Básicamente, la meta de la AC radica en ayudar al cliente a tomar contacto con las fuentes de recompensa que se han truncado, por la razón que sea. Como consecuencia de recuperar ese contacto, el cliente saldrá de su situación depresiva. Aunque se trabaja a partir de las conductas que tienen un efecto reforzante -y que no son evitaciones- de fondo están siempre los valores identificados, de manera que sean estos los que ayuden a la elección de las conductas a activar. La AC puede parecer un tratamiento sencillo (y lo es en sus técnicas), pero requiere de una comprensión en profundidad de los principios conductuales y exige del clínico durante toda la intervención una perspectiva funcional.

Martell et al. (2010, pp. 22-34) ofrecen unos principios esenciales en los que se asienta la intervención. Son las claves que el psicólogo debe tener presentes en todas y cada una de las sesiones en que trabaje desde la AC. De alguna manera, mientras se cumple este decálogo, la conducta del clínico es identificable como propia de la AC. Estos puntos son los siguientes: 
1. La clave para cambiar cómo se sienten las personas estriba en ayudarles a cambiar lo que hacen.

2. Distintos cambios vitales pueden llevar a la depresión, y las estrategias inmediatas que se emplean para afrontarlos pueden bloquear indefinidamente a las personas.

3. La pista que ayuda a averiguar qué será antidepresivo para un cliente en particular estriba en saber qué precede y qué sigue a las conductas importantes.

4. Hay que estructurar y programar actividades de acuerdo con un plan, no con un estado de ánimo.

5. El cambio resultará más fácil si se empieza muy poco a poco.

6. Deben priorizarse las actividades que tendrán un reforzamiento natural.

7. El terapeuta debe actuar como un entrenador.

8. Hay que insistir en mantener una aproximación empírica de solución de problemas, y reconocer que cualquier resultado puede ser útil.

9. No solo hables: jactúa!

10. El terapeuta debe trabajar para solucionar las barreras actuales a la activación o las que posiblemente aparecerán.

De acuerdo con la propuesta recogida en el manual en español más recientemente publicado (Barraca \& Pérez-Álvarez, 2015, pp. 113123) las intervenciones propugnadas desde los modelos de Martel et al. (2010) y Lejuez, Hopko, Acierno, Daughters y Pagoto (2011) puede integrarse en una secuencia común que conjugue las mejores aportaciones de ambos enfoques. En las siguientes líneas, y de forma esquemática se recorre este formato integral de intervención:

\section{Fase de Evaluación}

1. Tras los contactos iniciales, en los que se empleará ya el estilo colaborativo propio de la terapia, se genera confianza, se procura poner las bases para una buena alianza, y se ofrece una información clara y precisa sobre la depresión y la dinámica general de este tratamiento, comienza propiamente la fase de evaluación, donde, a la lógica recolección de manifestaciones depresivas a través de la entrevista, se añade un análisis del entorno y su pérdida potencial de capacidad reforzante. Se tienen en cuenta las actividades que se están llevando a cabo, las que se han abandonado, las rutinas perdidas y el papel de familiares y amigos (en particular, la posibilidad de que estén, sin advertirlo, reforzando las conductas que perpetúan la depresión). Se valora el proceso -ya gradual, ya progresivo- que pudo conducir a la depresión y la situación actual de reforzamiento general del entorno.

2. En cada sesión y durante toda la terapia, terapeuta y cliente organizan el tiempo de su encuentro a partir de la agenda, que decidirán en los primeros minutos de la consulta.

3. El terapeuta indica la necesidad de que el cliente se monitorice por medio del registro diario de sus actividades y del estado de ánimo (o la emoción) asociado a estas. El registro provee al clínico de una línea base para comparar el nivel de actividad (y su reforzamiento asociado) al inicio de la terapia y tras su finalización y sirve para el establecimiento de los 
objetivos terapéuticos, por lo que el terapeuta intentará hacer ver al cliente que su cumplimiento resulta fundamental.

4. Como apoyo en esta recogida de datos el terapeuta podrá solicitar que se complete algunos cuestionarios empleados en esta intervención, como el Behavioral Activation for Depression Scale - BADS (Kanter, Mulick, Busch, Berlin, \& Martell, 2007), el Environmental Reward Observation Scale - EROS (Armento \& Hopko, 2007), el Reward Probability Index RPI (Carvalho et al., 2011) o el Beck Depression Inventory-II - BDI-II (Beck, Steer y Brown, 1996).

5. Con toda la información se elabora un modelo explicativo, a través del análisis funcional, que justifique el trabajo a lo largo del resto de la terapia. A partir de ese análisis se transmite más fácilmente la racionalidad del tratamiento, pues se dilucida la vinculación entre actividades y bienestar, al igual que la relación entre aislamiento o evitación y empeoramiento del estado anímico.

\section{Fase de establecimiento de objetivos}

1. Con los datos y el análisis funcional, el terapeuta y el cliente pueden establecer los objetivos terapéuticos. Esta fase se entiende como una colaboración entre ambos y en la que debe mantenerse un enfoque idiográfico. En este momento se procura determinar cuáles son las conductas objetivo (aquellas que, por su capacidad reforzante natural deben aparecer o incrementarse) y las conductas problemáticas (aquellas que, por tratarse de conductas de evitación o insanas, deben extinguirse), cuáles las metas perseguidas y qué elementos o actividades pueden funcionar como recompensas previas, si se considera necesario para aumentar la motivación al inicio de la intervención.

2. Como apoyo en este proceso se procura crear un entorno saludable, en el que familiares y amigos sean informados de la necesidad de no centrarse -no reforzar- los síntomas depresivos sino, al contrario, de ayudar al sujeto a involucrarse en alternativas más sanas y a la larga adaptadas.

3. Es imprescindible que el cliente entienda y comparta la racionalidad del tratamiento, pues solo así se movilizará. Básicamente debe trasmitírsele que, sea cual sea la razón por la que se deprimió, el camino para librarse de los pensamientos negativos, la pasividad, la tristeza, las emociones dolorosas, etc. no es hablar de su posible causa, sino adoptar una posición activa $y$, por medio de la realización de determinadas actividades, entrar en contacto con situaciones que le gratificarán y que se corresponden con las cosas que realmente le importan y quiere en su vida. Aunque el cliente crea que actuar así es muy difícil si no gana primero más energía o ánimo, se le explica que el planteamiento es justo al revés: logrará esa energía según se ponga en marcha; pero no debe agobiarse: el avance o las exigencias serán progresivas, estarán siempre a su alcance, $y$, además, contará en todo momento con la ayuda del terapeuta, que actuará como un entrenador, por lo que le estimulará y le ofrecerá soluciones si surgen dificultades. Si el caso es el de un cliente deprimido que sí está activo, pero su laboriosidad está al servicio de la evitación, entonces la propuesta incidirá en tener presentes las cosas que le importan en su vida 
y dejar de evitar las tareas fundamentales, de forma que la sensación de gratificación y de deber cumplido puedan alcanzarse. La idea de la AC no es hacer más actividades, sino llevar a cabo aquellas que dan sentido a la vida.

4. Asociada a esta comprensión del modelo de intervención se encuentra la necesidad de comprometerse hasta la finalización del tratamiento. Si el cliente deja de venir tras unas sesiones, no puede garantizarse que la depresión no reaparezca y, muy posiblemente, con mayor virulencia. Conviene mencionar que, aunque algunos días se sienta desmotivado, triste, cansado y no le vea sentido a la terapia (lo que muy probablemente ocurrirá), hará bien en acudir, pues posiblemente esta situación mejorará al recibir atención y esa actitud general de seguir el plan no obstante el bajo estado de ánimo justamente es la que le ayudará a salir de la situación depresiva.

5. Se delimitan entonces los objetivos de la intervención. Una alternativa si las metas no están claras, consiste en aprovechar los listados de áreas vitales. Se pueden extraer ideas de la clasificación de Hayes, Strosahl y Wilson (1999) o de Wilson, Sandoz, Kitchens y Roberts (2010). Otra opción estructurada y práctica es la de aprovechar la última clasificación de Lejuez et al. (2011) y apuntar en un registro, dentro de sus cinco áreas globales qué valores son los más importantes para uno.

6. Decididas las áreas vitales donde hay que incidir y los distintos valores (u objetivos) dentro de ellas, llega el momento de determinar las conductas concretas que dirigirán a esos objetivos y programar su realización para los siguientes días. De cara a la programación fu- tura, conviene jerarquizar las actividades para que la incorporación que se prevé esté ya organizada, aunque lógicamente podrá variar según las circunstancias.

Fase de intervención: incorporación de las técnicas de la AC

1. Cuando se inicia el tratamiento es importante contar con un entorno estructurado, adecuadamente directivo y capaz de dar un apoyo conveniente al cliente. No obstante, según avance el proceso, la guía y los apoyos se desvanecerán. Por eso, es habitual empezar la terapia con un par de consultas semanales (especialmente, con los pacientes más deprimidos) y luego mantener, aproximadamente, una sesión a la semana. Reforzar socialmente el cumplimiento de las prescripciones y la consecución de las metas es una tarea básica del terapeuta que debe mantener a lo largo de toda la intervención.

2. Aclarada y compartida la necesidad de llevar a cabo las tareas seleccionadas, en diálogo con el cliente, el terapeuta apuntará en sucesivas hojas de registro (las mismas que sirvieron para monitorizar el estado de ánimo) aquellas actividades que deben llevarse a cabo antes de la siguiente sesión.

3. Como la incorporación de actividades debe jerarquizarse, de menor a mayor dificultad, hay que decidir cuáles y cuántas actividades se incorporan cada semana. Un criterio contrastado apunta que entre una y tres actividades semanales es un número adecuado. Así, aproximadamente tras unos dos meses de tratamiento se pueden haber incorporado unas 15 o 16 actividades, que es una cantidad 
que permitiría finalizar la intervención con garantías. No obstante, el número concreto de actividades por semana y al final de la intervención depende de cada caso. Lejuez et al. (2001) mencionan que la primera semana basta con una o dos y que en semanas posteriores pueden incorporarse hasta cinco. De acuerdo con el programa de Martell et al. (2010) no hay que fijar ningún número específico por semana, pero sí que es importante hacer una elección progresiva y acorde con la capacidad de activación del sujeto en cada momento. En cualquier caso, si los objetivos semanales fueron sobreestimados, pueden corregirse para disminuirse; y también cabe lo contrario: incrementarlos en número o dificultad si resultaron excesivamente sencillos y el cliente se recupera a un ritmo más rápido de lo esperado.

4. Respecto a las actividades que escoger, lo adecuado es que cubran un amplio rango. De este modo, se plantearán desde actividades sencillas y que lleven poco tiempo (para las primeras semanas) hasta otras más extensas en tiempo y más difíciles (para las últimas del tratamiento). Hay que recordar que las conductas de evitación tienen un reforzamiento (negativo) inmediato y seguro, mientras que las de activación con las que entran en competencia pueden tardar en recompensar. Por esta razón, emplear en las primeras semanas tareas fáciles cuyo reforzamiento es rápido y seguro, actividades que, al momento o poco después de realizadas, generen una sensación grata y de "deber cumplido" resulta lo más conveniente. Además, optar por aquellas que supongan una movilización corporal es probablemente lo mejor: está bien establecido que la prácti- ca diaria de actividades físicas tiene un efecto antidepresivo; aunque, naturalmente, siempre hay que considerar el nivel de actividad previo y el estado físico del cliente. Igualmente, si por la depresión se han abandonado actividades rutinarias y básicas (por ejemplo, cuidar la higiene personal, vestirse, salir de casa, comer tres veces al día, acostarse y levantarse a horas adecuadas, etc.) estas también son buenas candidatas para figurar en las hojas de actividades de las primeras semanas. Una vez dados estos primeros pasos, otras tareas de activación resultarán más sencillas, pues la actitud pasiva y letárgica genera más cansancio y más dificultad para enfrentarse a las tareas, mientras que la actividad llama a la actividad.

5. Mientras se incorporan en cada consulta nuevas actividades en el registro, las de las semanas anteriores siguen haciéndose y se integran como rutinas de forma que, progresivamente, hay un conjunto mayor de actividades que proporcionan reforzamiento natural. Como las primeras actividades (más sencillas) están ya proporcionando reforzamiento, pueden añadirse otras cuyas consecuencias gratificantes tardarán en llegar pero que a la larga también serán fuente de reforzamiento.

6. Si se traen a consulta los registros de las actividades programadas, a través de su revisión se puede comprobar el cumplimiento diario. En general, llevar el control de las actividades que se realizaron y del estado de ánimo asociado es una buena forma para identificar los factores contextuales y el papel de la propia actividad en la depresión, de forma que, a la larga, se garantice el mantenimiento de los beneficios terapéuticos. 
7. A partir del momento en que se empiezan a incluir las tareas de activación se revisan en todas las sesiones y se indaga sobre su cumplimiento y las dificultades surgidas. En el caso de que las tareas no se realicen o se hagan solo parcialmente, el terapeuta adoptará una actitud de solución de problemas y se orientará hacia la corrección de esta circunstancia. Decidida la manera de afrontarla, se volverán a poner en la hoja de actividades con los cambios pertinentes para garantizarlas, o se incluirán unas conductas previas para facilitarlas, 0 se sustituirán por otras, si se estima que es lo mejor. Si el cumplimiento fue el esperado, se añaden las correspondientes a las programadas para la siguiente semana. Es importante mantener constantemente una actitud positiva y alentadora. El cumplimiento parcial de las actividades es muy corriente en los clientes deprimidos. Por eso, el terapeuta apreciará siempre el esfuerzo y reforzará todo lo que suponga un incremento por encima de la línea base.

8. Una posible ayuda que el terapeuta puede valorar incluir para lograr el cumplimiento de las actividades -sobre todo si aprecia que las tareas se le hacen muy cuesta arriba al cliente- consiste en reforzar positivamente de forma directa las conductas antidepresivas, siempre que se han alcanzado al final de la semana los objetivos planteados. Contar con un premio por el esfuerzo que se ha llevado a cabo durante una semana favorece mantenerse en la tarea. Por tanto, si se han cumplido las actividades programadas en la semana y durante el tiempo suficiente, el cliente podrá otorgarse un reforzador previamente establecido. Por supuesto, los reforzadores no deben suponer nunca un apoyo de las conductas depresivas.

9. Junto con este autoreforzamiento también puede añadirse el reforzamiento y ayuda que otorgan familiares o amigos y que se hayan estipulado previamente en contratos conductuales. Este método tiene especial cabida cuando el terapeuta detecta la necesidad de desarrollar un ambiente que no refuerce las conductas depresivas y el cliente, por sí solo, tiene dificultad para cumplir con las conductas comprometidas.

10. Mientras sigue el proceso de activación semana a semana, tal y como se acaba de describir, el terapeuta se mantendrá muy atento al efecto que esta incorporación de actividades provoca en el ánimo del sujeto, y le preguntará sobre el particular. Si todo transcurre sin problemas porque se han elegido conductas adecuadas y se han regulado convenientemente, el cliente mejorará, mostrará poco a poco mayor energía y bienestar; las puntuaciones del BDI-Il o el EROS corroborarán este progreso; el número de actividades desplegadas será cada vez mayor; y es probable que el cliente manifieste verbalmente su mejoría y su satisfacción por los cambios acaecidos. Pero si esto no sucede, si, a pesar de llevar a cabo las actividades no avanza, el terapeuta animará al cliente a perseverar y comenzará un proceso de indagación y resolución de problemas para descubrir qué factores impiden el progreso. Debe preguntarse entonces si las actividades están bien reguladas en su nivel de dificultad y en su cuantía, si se están asociadas a valores importantes del sujeto, si se produce interferencia por las conductas de evitación, o si el pensamiento rumia- 
tivo está impidiendo el contacto con los reforzadores, por citar los problemas más comunes.

11. A la par que el proceso de activación se habrá previsto la extinción de las conductas de evitación, si estas habían sido identificadas. Es probable que la misma programación de determinadas conductas contrarreste la aparición de las de evitación, pero no siempre es así. Es en este último caso cuando se abordan directamente. Si un sujeto presenta conductas de evitación tales como levantarse tarde y quedarse amodorrado en la cama casi toda la mañana y, además, ver varias horas programas insustanciales en la televisión durante la tarde, el terapeuta puede proponer como conductas de activación dar un paseo vigoroso por la mañana y quedar con un amigo por la tarde. Estas acciones representan tanto una activación directa como la eliminación de conductas de evitación. En cambio, si un sujeto lleva a cabo muchas tareas (por ejemplo, sale y se encuentra con mucha gente, hace deporte agotador durante horas y horas, se pone a ayudar a sus vecinos en arreglos sencillos), pero todas ellas están al servicio de evitar actividades que realmente debe acometer (escribir un informe difícil de su trabajo, estudiar un examen que le agobia, arreglar temas pendientes con su ex mujer, etc.) el terapeuta debe plantear una eliminación o disminución progresiva de las conductas de evitación y no sustituirlas por otras, sino que se dejen de postergar las importantes (redactar el informe, estudiar el examen, hablar con su mujer, etc.). El terapeuta ayudará a que se afronten con garantías: facilitándolas por medio de ensayos previos, de guiones, dividiéndolas en pasos sencillos, etc.
12. Aunque se decidieran al principio unas actividades concretas es muy posible que, al avanzar el tratamiento, se descubra que algunas de ellas no son las más adecuadas. Según se progresa, el cliente puede cambiar de opinión, ver las cosas de otra manera y ampliar su plan (o, al contrario, desear aminorarlo). Nada proscribe estos cambios. Es más, el terapeuta preguntará en las distintas sesiones si piensa que las actividades elegidas siguen siendo las mejores y si continúan respondiendo a sus valores. Si el cliente ya nos las ve útiles se buscarán otras que supongan una mejor alternativa. También es adecuado revisar el reforzamiento arbitrario directo o los contratos establecidos por si se decide repetirlos, cambiarlos o abandonarlos porque ya hayan cumplido su función.

13. Si el cliente no obtiene los beneficios esperables a pesar de que lleva a cabo las actividades durante un tiempo suficiente, ha extinguido sus conductas de evitación y ha incluido conductas que realmente se corresponden con sus valores, el terapeuta debe investigar si la falta de resultados es fruto de la interferencia de pensamientos repetitivos y auto-críticos. Entonces puede plantear al cliente que las actividades están haciéndose "solo con el cuerpo, pero no con la mente"; es decir, sin poner atención y cuestionando internamente el sentido de hacerlo y su utilidad, o comparándolo con veces del pasado, o con la diligencia de otras personas, etc. Si este es el caso, en este momento de la intervención deben ponerse en marcha las estrategias para volver a "contactar con la experiencia".

14. Simultánea o alternativamente pueden darse también otras circunstancias que supon- 
gan trabas para conseguir el reforzamiento de las actividades. Por ejemplo: problemas derivados de la carencia de habilidades sociales, dificultades surgidas por la ansiedad, reacciones emocionales muy intesificadas que forman parte de la historia de aprendizaje y que están condicionadas a determinadas situaciones, etc. De nuevo con una metodología de solución de problemas, y en colaboración con el cliente, el terapeuta tratará de sortear estos obstáculos empleando las técnicas pertinentes (relajación, ensayos mentales de las tareas asignadas, role-playing, entrenamiento en habilidades sociales específicas, etc.), de forma que se recupere el camino hacia el reforzamiento de la actividad.

15. Finalmente, la última parte de la terapia, una vez que tanto indicadores subjetivos (impresión del propio cliente, del terapeuta, de otras personas allegadas, etc.), como objetivos (número de actividades realizadas, ausencia de evitaciones, atención mantenida en las tareas, puntuaciones en los cuestionarios, alta laboral, retirada de la medicación, etc.) demuestren la recuperación y la estabilidad anímica, el terapeuta propondrá unas sesiones dedicadas al cierre de la terapia y a la prevención de recaídas. Finalmente, espaciará las sesiones y propondrá el necesario seguimiento.

\section{Solucionar problemas}

\section{de la puesta en práctica de la AC}

En una terapia como la $\mathrm{AC}$, en que por la programación de actividades hay una exigencia sobre los clientes, los problemas en la aplicación son corrientes. A la frustración, la desesperanza, la culpa, la abulia, la duda, la ira, el blo- queo, la confusión habituales en alguien deprimido se suma la dificultad para cumplir con las tareas encomendadas. El terapeuta debe estar preparado para que el cliente le diga que no ha cumplido nada de lo programado (o, peor aún, que mienta, por vergüenza, y le diga que sí lo ha cumplido), que piensa tirar la toalla (jo que ya lo ha hecho!) o que no alberga ya ninguna fe en la terapia, aunque al principio comentó que la tenía por su última esperanza. La labor de un terapeuta ante este escenario pasa por llevar a cabo una evaluación conductual que le ayude a identificar por qué han surgido las dificultades o se ha intensificado el malestar del cliente, y una vez detectados estos factores enfrente la situación como una tarea para resolver. En vez de culpar a los mismos clientes, tildarles de derrotistas, personas que no quieren dejarse ayudar $\mathrm{o}$ atribuir las dificultades a algún problema de personalidad, el terapeuta adoptará un enfoque de resolución de problemas y procurará "sacar lo mejor de sí mismo" (Martell et al., 2010, p. 153) para volver a esperanzar al cliente y encarrilarlo de nuevo hacia la mejoría.

Dadas las dificultades mencionadas y asociadas a la misma activación, es necesario tener presentes algunas claves generales que ayudarán siempre al afrontamiento de este tipo de problemas. Se enumeran a continuación recogiendo y sintetizado las propuestas de Barraca y Pérez-Álvarez (2015) y de Martell et al. (2010).

1. Comprender la racionalidad del tratamiento. Aunque durante un tiempo, por la razón que sea, se hayan abandono las tareas, si se ha comprendido la racionalidad del tratamiento será más probable recuperarlas. Al 
haber explicado previamente que es fácil que los efectos gratificantes tarden en aparecer, se motiva la ejecución de las acciones aunque no se obtengan cambios todavía. La persona deprimida debe hacer las tareas adecuadas aunque esté desmotivado y "no note nada", es más, incluso aunque al principio "se note peor", pues la activación es una intervención que necesita tiempo para resultar efectiva.

2. Mantener las sesiones con estructura, centradas en la resolución de problemas y enfocadas hacia la activación. Ese estilo es una fuente de apoyo tanto el terapeuta como para el cliente, y una guía para seguir adelante en los momentos más difíciles. Aunque es cierto que el cliente puede desear (y necesitar) en algún momento desahogarse y hablar de las tristes vicisitudes que ha tenido que soportar, tras un periodo razonable, el terapeuta debe volver a orientar la sesión hacia las actividades que prevendrán un bajo estado de ánimo.

3. Validar siempre los sentimientos y los comportamientos del cliente. Es normal que, cuando se ha incumplido lo comprometido, la persona se sienta especialmente mal y tema recriminaciones, más directas o más veladas, por parte del terapeuta. Pero si este, en cambio, comprende lo que sucede y así se lo transmite al cliente es mucho más probable que reúna las fuerzas para volver a intentarlo, especialmente si juntos buscan una forma más sencilla o segura de conseguir llevar a cabo la asignación.

4. Recordar el principio de que "todo puede valer". Saber qué ha fallado es importante para preparar un plan mejor, con más ayudas o con más detalle. Si el cliente ve esta actitud por parte de su terapeuta y no una actitud de culparle ante el fracaso, seguramente se relajará y se volverá a motivar. Si en la AC el mayor peso del comportamiento se atribuye a las contingencias ambientales, coherentemente con este principio el terapeuta debe aprender de esas contingencias y aprovecharlas a su favor. De esta forma, se desecharán siempre atribuciones para el incumplimiento como "el autosabotaje", "la vangancia", "la intolerancia a la frustración”, “los problemas del Eje II”, etc. que ponen todo el foco en el interior del sujeto o en sus rasgos de personalidad.

5. Mantener el optimismo y la persistencia. El terapeuta de la AC debe recordar que si trabaja con una mentalidad abierta y busca las claves que controlan la conducta, la situación del cliente mejorará. No debe perder esa perspectiva, a pesar de que por momentos haya vueltas atrás. Además, si el clínico sigue comprometido con estas actitudes a pesar de los fracasos, servirá como modelo positivo para el cliente. Le transmitirá que, si persiste, lo logrará.

6. Por último, es conveniente mantener una actitud previsora, que anticipe los problemas que en determinados momentos pueden surgir cuando se planifican tareas a una semana vista. Así, por ejemplo, empezar a programar el correr por la calle o por el campo cuando empieza el frío y la lluvia, o la realización de determinadas tareas en casa cuando es Navidad y van a venir unos parientes, son muestras de falta de previsión por parte del terapeuta.

\section{Variantes en la aplicación de la AC}

Distintos trabajos han demostrado ya la eficacia de utilizar la AC, sola o en combinación 
con otros tratamientos, para ayudar a personas con problemas diferentes de la depresión unipolar. Para empezar, los principios y procedimientos de la AC han servido para tratar la distimia y el trastorno bipolar, y también cuadros mixtos ansioso-depresivos. Así mismo, la $A C$ se ha utilizado en situaciones de comorbilidad de depresión con otros trastornos o enfermedades, como el cáncer, el dolor crónico, la obesidad, la dependencia de sustancias o el trastorno límite de la personalidad. Pero junto con estas variantes relacionadas con distintos problemas, la AC se ha adaptado para formatos grupales y para aplicaciones en distintas edades, desde adolescentes a ancianos, y poblaciones (distintas razas, culturas y etnias) y diferentes ámbitos (rural, aplicación online, etc.).

Respecto a las variantes de la depresión y la ansiedad, distintos trabajos han revelado que la intervención de $A C$ ha servido para tratar casos en que la depresión se combinaba con síntomas de ansiedad (Barraca, 2010; Hopko, Lejuez, \& Hopko, 2004). Si se ha planteado el uso de la $A C$ en situaciones que mezclen ambos problemas se debe a la similitud funcional que - de acuerdo con el mismo modelo de la AC- puede darse entre la depresión y la ansiedad. Tradicionalmente, la perpetuación de la ansiedad se ha explicado por las conductas de evitación y, justo por eso, la exposición (en la modalidad que conviniese) se ha tenido por el tratamiento más recomendable. Pero para la AC la depresión también puede mantenerse por las conductas de evitación y, por tanto, cuando la activación se prescribe para dejar de evitar situaciones incómodas o dolorosas a corto plazo, se sigue la misma lógica que en el tratamiento tradicional de la ansiedad. Si las terapias de exposición han mostrado un éxito tan notable en las fobias, el trastorno de angustia, el trastorno obsesivo-compulsivo, el trastorno de estrés postraumático, etc. por los componentes evitativos que retaban, es lógico que tenga igualmente eficacia en la depresión y que la terapia de una persona con ambos cuadros gane en eficiencia por las sinergias del tratamiento.

Turner y Leach (2010), han defendido que la ansiedad comparte funcionalidad con la depresión porque las personas con la primera muestra una alta frecuencia de reforzamiento negativo, que es tan característico de las conductas evitativas. Defienden que la AC puede ser tan efectiva en el tratamiento de la ansiedad como lo es en la depresión. Y, así, presentan una series de casos en personas con cuadros exclusivamente ansiosos en el que una intervención de activación conductual -que denominan BATA: Behavioral Activation Treatment of Anxiey (Tratamiento de Activación Conductual de la Ansiedad)-y que incluye monitorización, establecimiento de objetivos, programación de actividades y su revisión, y un enfoque de solución de problemas colaborativo, resulta eficaz para mejorar la ansiedad sin el concurso de otros componentes tradicionales en el tratamiento de esta, como la exposición gradual, el entrenamiento en relajación o la reestructuración cognitiva.

El trastorno de estrés postraumático (TEP) es otro diagnóstico con un componente importante de ansiedad en el que se ha aplicado frecuentemente la AC (Cf. Gros, 2012; Jakupcak et al., 2006; Jakupcak, Wagner, Paulson, Varra, 
\& McFall, 2010; Mulick \& Naugle, 2010; Plagge, Lu, Lovejoy, Karl, \& Dobscha, 2013). En este cuadro la evitación guarda relación con los recuerdos traumáticos de la experiencia vivida, lo que, a su vez, puede favorecer el que esta siga presente durante años alterando gravemente la vida diaria y las actividades que se desarrollan. De hecho, es característico de las personas con TEP que se aíslen y que no se sientan capaces de hacer frente a su ansiedad. La AC aquí aplicada trata de recuperar esas actividades que dejaron de llevarse a cabo, aunque eso suponga volver a contactar, en un primer momento, con las experiencias dolorosas. De este modo, en la intervención se identifican las conductas de evitación actuales, así como las recompensas y objetivos terapéuticos que se alcanzarían si se extinguieran. En conjunto, el tratamiento con la AC ha mostrado en la mayoría de las investigaciones una mejora general en la calidad de vida y una reducción en los síntomas del TEP, que, de hecho, descendieron en mayor medida que los depresivos.

La versión breve de la AC (la correspondiente a Lejuez et al., 2001) se ha aplicado a enfermos oncológicos desde su misma aparición, y son habituales los estudios en que se ha incorporado, especialmente en pacientes con cáncer de mama (Cf. Armento \& Hopko, 2009; Hopko et al., 2011; Hopko et al., 2015; Ryba, Lejuez, \& Hopko, 2014). Esta asociación no resulta nada extraña, pues la depresión es uno de los problemas más vinculados a la enfermedad tumoral. Por otro lado, se ha demostrado la relación entre el deterioro de las actividades recreacionales y físicas, los problemas en las relaciones sociales, y la afectación del sueño con un progreso más rápido de los síntomas del cáncer, más probabilidades de metástasis, mayor dolor $y$, posiblemente, mayor mortalidad. Y en el dolor crónico, un problema en ocasiones asociado al cáncer, aunque también independiente, igualmente existen aplicaciones del tratamiento de la AC (Lundervold, Talley, \& Buermann, 2008; Plagge et al., 2013).

En conjunto, estos trabajos han demostrado la eficacia de esta modalidad de intervención y, sobre todo, su capacidad para integrarse adecuadamente en un tratamiento médico intensivo y que afecta severamente (intervenciones quirúrgicas, sesiones de quimioterapia o radioterapia, analíticas extensas y otras pruebas diagnósticas, revisiones, etc.); de hecho, su aplicación se ha producido fundamentalmente en entornos hospitalarios.

Existen publicaciones, ya más limitadas en su número pero ciertamente interesantes, de la aplicación de la AC a distintos problemas comportamentales. Entre estos, y sin la pretensión de ser exhaustivos, se encuentran los que muestran la utilidad de la $A C$ en personas con trastorno límite de la personalidad (Hopko, Sanchez, Hopko, Dvir, \& Lejuez, 2003), en pacientes con síntomas psicóticos (Romero Gamero, Poves Oñate, \& Vucínovich, 2011), consumo de drogas ilegales (Daughters et al., 2008), obesidad (Busch et al., 2013; Pagoto, 2008) o en fumadores con depresión moderada (McPherson et al., 2010).

Para concluir, debe mencionarse que la AC también ha cambiado de formato de aplicación para ajustarse a distintas necesidades. Así, existen adaptaciones a un formato grupal; para su aplicación tanto en población adolescente 
como en personas mayores; para clientes con niveles intelectuales o culturales bajos, distintas etnias o marcos culturales no occidentales y analfabetismo funcional (Cf. Barraca \& PérezÁlvarez, 2015 para una revisión de todas estas modalidades). Igualmente, se han desarrollado excelentes programas on-line para enseñar de la manera más práctica posible la lógica y la aplicación de la AC, especialmente dirigidos a la orientación de trabajadores sociales y mediadores en la búsqueda de empleo (Puspitasari, Kanter, Koerner, Murphy, \& Crowe, 2013).

\section{Conclusiones}

La depresión es uno de los problemas de salud mental más importantes con el que se enfrentan las sociedades contemporáneas. Un cuadro que parece crecer progresivamente. Es, por consiguiente, una situación a la que los psicólogos clínicos van a tener que hacer frente. En esta tesitura, saber que hay terapias psicológicas tan sencillas y tan eficaces como la AC que presentan mejores resultados en muchos casos que los antidepresivos y que además son mejor aceptadas por los pacientes (Craven \& Bland, 2006), más económicas a la larga (Dobson et al., 2008) y fáciles de implementar representa una gran ventaja y prepara para ofrecer un importante servicio a la sociedad.

Pero, junto a esto, no está de más señalar ahora que otra de las ventajas de conocer y aplicar la AC estriba en favorecer una perspectiva de la depresión genuinamente psicológica, que vuelve a situar su génesis y su mantenimiento en los avatares vitales que experimenta el sujeto y en sus propios comportamientos; que no ve a quien la experimenta preso de un déficit (cognitivo, neurológico, químico o cualquier otro); y que supone también liberarla de las concepciones biologicistas que imperan en la actualidad y que conciben este cuadro no como una manifestación de problemas vitales sino como una enfermedad (Barraca, 2009). En suma, cambiar la concepción sobre la depresión implica desmedicalizar su tratamiento (Pérez-Álvarez, 2007), volver a dotar al sujeto de responsabilidad activa $y$, a la larga, prevenir esa expansión de carácter casi epidémico.

Sin embargo, para que esto pueda convertirse en una realidad la $A C$ debe aún seguir un camino que incluye el mantener su vigor investigador para continuar aportando pruebas de su eficacia frente a otros tratamientos (como el cognitivo o la medicación), conseguir una mayor divulgación y ofrecer una imagen más integrada. Trabajos como el contenido en estas páginas son un intento por facilitar esa divulgación y aclarar las dudas respecto a su aplicación mas adecuada.

\section{Referencias}

American Pyschology Association (APA), Division 12. (2015). Depression. Recuperado de: https://www.div12.org/psychologicaltreatments/disorders/depression/

Armento, M. E. A., \& Hopko, D. R. (2007). The Environmental Reward Observation Scale (EROS): Development, validity, and reliability. Behavior Therapy, 38, 107-119.

Armento, M. E. A., \& Hopko, D. R. (2009). Behavioral Activation of a breast cancer patient with coexistent major depression and generalized anxiety disorder. Clinical Case Studies, 8, 25-37.

Barraca, J. (2009). La Activación Conductual (AC) y la Terapia de Activación Conductual para la Depresión (TACD): dos protocolos de tratamiento desde el modelo de la activación conductual. EduPsykhé, 8, 23-47. 
Barraca, J. (2010). Aplicación de la Activación Conductual en un paciente con sintomatología depresiva. Clínica y Salud, 21, 183-197.

Barraca, J., \& Pérez-Álvarez (2015). Activación Conductual para el tratamiento de la depresión. Madrid: Síntesis.

Beck, A. T., Rush, A. J., Shaw, B. F., \& Emery, G. (1979). Cognitive therapy of depression. New York: Guilford.

Beck, A. T., Steer, R. A., \& Brown, G. K. (1996). Manual for the Beck Depression Inventory-II. San Antonio, TX: Psychological Corporation. Busch, A. M., Whited, M. C., Appelhans, B. M., Schneider, K. L., Waring, M. E., DeBiasse, M. A.,... Pagoto, S. L. (2013). Reliable change in depression during behavioral weight loss treatment among women with major depression. Obesity, 21, E211-8. doi: 10.1002/oby.20113

Carvalho, J. P., Gawrysiak, M. J., Hellmuth, J. C., McNulty, J. K., Magidson, J. F., Lejuez, C. W., \& Hopko, D. R. (2011). The Reward Probability Index: Design and validation of a scale measuring access to environmental reward. Behavior Therapy, 42, 249-262.

Craven, M. A., \& Bland, R. (2006). Better practices in collaborative mental health care: An analysis of the effective base. Canadian Journal of Psychiatry, 51 (Suppl. 1), 7S-72S.

Daughters, S. B., Braun, A. R., Sargeant, M. N., Reynolds, E. K., Hopko, D. R., Blanco, C., \& Lejuez, C. (2008). Effectiveness of a brief behavioral treatment for inner-city illicit drug users with elevated depressive symptoms: The Life Enhancement Treatment for Substance Use (LETS Act!). Journal of Clinical Psychiatry, 69, 122-129.

DeRubeis, R. J., \& Crits-Christoph, P. (1998). Empirically supported individual and group psychological treatments for adult mental disorders. Journal of Consulting and Clinical Psychology, 66, 37-52.

Dobson, K. S., Hollon, S. D., Dimidjian, S., Schmaling, K. B., Kohlenberg, R. J., Gallop, R.,... Jacobson, N. S. (2008). Randomized trial of Behavioral Activation, Cognitive Therapy, and antidepressant medication in the prevention relapse and recurrence in major depression. Journal of Consulting and Clinical Psychology, 76, 468-477.

Ferster, C. B. (1973). A functional analysis of depression. American Psychologist, 28, 850870.

Gortner, E. T., Gollan, J. K., Dobson, K. S., \& Jacobson, N. S. (1998). Cognitive-behavioral treatment for depression: Relapse prevention. Journal of Consulting and Clinical Psychology, 66, 377-384.

Gros, D. F., Price, M., Strachan, M., Yuen, E. K., Milanak, M. E., \& Acierno, R. (2012). Behavioral activation and therapeutic exposure: An investigation of relative symptom changes in PTSD and depression during the course of integrated behavioral activation, situational exposure, and imaginal exposure techniques. Behavior Modification, 36, 580-599.

Hayes, S. C., Strosahl, K. D., \& Wilson, K. G. (1999). Acceptance and commitment therapy: An experiential approach to behavior change. New York: Guilford.

Hopko, D. R., Armento, M. E. A., Robertson, S., Ryba, M. M., Carvalho, J. P., Colman, L. K.,...Lejuez, C. W. (2011). Brief behavioral activation and problem-solving therapy for depressed breast cancer patients: Randomized trial. Journal of Consulting and Clinical Psychology, 79, 834-849.

Hopko, D. R., Cannity, K., McIndoo, C. C., File, A. A., Ryba, M. M., Clark, C. G., \& Bell, J. L. (2015). Behavior therapy for depressed breast cancer patients: Predictors of treatment outcome. Journal of Consulting and Clinical Psychology, 83, 225-231

Hopko, D. R., Lejuez, C., \& Hopko, S. D. (2004). Behavioral activation as an intervention for coexistent depressive and anxiety symptoms. Clinical Case Studies, 3, 37-48.

Hopko, D. R., Lejuez, C. W., Ruggiero, K. J., \& Eifert, G.H. (2003). Contemporary behavioral activation treatment for depression: Procedures, principles, and progress. Clinical Psychology Review, 23, 699-717.

Hopko, D., Sanchez, L., Hopko, S., Dvir, S., \& Lejuez, C. (2003). Behavioral activation and the prevention of suicidal behaviors in patients with borderline personality 
disorder. Journal of Personality Disorders, 17, 460-478.

Jacobson, N. S., Dobson, K. S. Truax, P. A., Addis, M. E., Koerner, K., Gollan, J. K.,... Prince, S. E. (1996). A component analysis of cognitivebehavioral treatment for depression. Journal of Consulting and Clinical Psychology, 64, 295-304.

Jacobson, N. S., Martell, C. R., \& Dimidjian, S. (2001). Behavioral activation treatment for depression: Returning to contextual roots. Clinical Psychology: Science and Practice, 8, 255-270.

Jakupcak, M., Roberts, L. J., Martell, C., Mulick, P., Michael, S., Reed, R.,... McFall, M. (2006). A pilot study of behavioral activation for veterans with posttraumatic stress disorder. Journal of Traumatic Stress, 19, 387-391.

Jakupcak, M., Wagner, A., Paulson, A., Varra, A., \& McFall, M. (2010). Behavioral activation as a primary care-based treatment for PTSD and depression among returning veterans. Journal of Traumatic Stress, 23, 491-495.

Kanter, J. W., Mulick, P. S., Busch, A. M., Berlin, K. S. y Martell, C. R. (2007). The Behavioral Activation for Depression Scale (BADS): Psychometric properties and factor structure. Journal of Psychopathology and Behavior Assessment, 29, 191-202.

Lejuez, C.W., Hopko, D. R, Acierno, R., Daughters, S. B, \& Pagoto, S. L. (2011). Ten year revision of the brief behavioral activation treatment for depression: revised treatment manual. Behavior Modification, 35, 111-161.

Lejuez, C. W., Hopko, D. R., \& Hopko, S. D. (2001). A brief Behavioral Activation Treatment for Depression: Treatment manual. Behavior Modification, 25, 225-286.

Lejuez, C. W., Hopko, D. R., LePage, J., Hopko, S. D., \& McNeil, D. W. (2001). A brief Behavioral Activation Treatment for Depression. Cognitive and Behavioral Practice, 8, 164175.

Lewinsohn, P. M. (1974). A behavioral approach to depression. En R. M. Friedman, \& M. M. Katz (Eds.), The psychology of depression: Contemporary theory and research (pp. 157185). New York: John Wiley.
Lundervold, D., Talley, C., \&Buermann, M. (2008). Effect behavioral of behavioral activation treatment on chronic fibromyalgia pain: replication and extension. International Journal of Behavioral Consultation and Therapy, 4, 146-157.

MacPherson, L., Tull, M. T., Matusiewicz, A. K., Rodman, S., Strong, D. R., Kahler, C. W., \& Lejuez, C. W. (2010). Randomized controlled trial of behavioral activation smoking cessation treatment for smokers with elevated depressive symptoms. Journal of Consulting and Clinical Psychology, 78, 55-61.

Martell, C. R. (2015). Behavioral Activation Therapy. Recuperado de http://www. christophermartell.com/ba.php

Martell, C. R., Addis, M. E., \& Jacobson, N. S. (2001). Depression in context: Strategies for guided action. New York: W. W. Norton.

Martell, C. R., Dimidjian, S., \& Herman-Dunn, R. (2010). Behavioral Activation for Depression: A Clinician's Guide. New York: Guilford.

Mulick, P. S., \& Naugle, A. E. (2010). Behavioral activation in the treatment of comorbid posttraumatic stress disorder and major depressive disorder. International Journal of Behavioral Consultation and Therapy, 5, 330350.

Pagoto, S., Bodenlos, J. S., Schneider, K. L., Olendzki, B., Spates, C. R., \& Ma, Y. (2008). Initial investigation of behavioral activation therapy for co-morbid major depressive disorder and obesity. Psychotherapy: Theory, Research, Practice, Training, 45, 410-415.

Pérez-Álvarez, M. (2007). La Activación Conductual y la desmedicalización de la depresión. Papeles del Psicólogo, 28, 97-110.

Plagge, J. M., Lu, M. W., Lovejoy, T. I., Karl, A. I., \& Dobscha, S. K. (2013). Treatment of comorbid pain and PTSD in returning veterans: A collaborative approach utilizing behavioral activation. Pain Medicine, 14, 1164-1172.

Puspitasari, A., Kanter, J., Koerner, K., Murphy, J., \& Crowe, A. (2013). Developing an online, modular, active learning training program for Behavioral Activation. Psychotherapy, 50, 256-265. 
Romero Gamero, R., Poves Oñate, S., \& Vucínovich, N. (2011). Terapia de activación conductual para la depresión aplicación a un paciente con esquizofrenia paranoide. Análisis y Modificación de Conducta, 37, 6575.

Ryba, M. M., Lejuez, C. W., \& Hopko, D. R. (2014). Behavioral activation for depressed breast cancer patients: The impact of therapeutic compliance and quantity of activities completed on symptom reduction. Journal of Consulting and Clinical Psychology, 82, 325-335.

Turner, J. S., \& Leach, D. J. (2010). Experimental evaluation of behavioral activation treatment of anxiety (BATA) in three older adults. International Journal of Behavioral Consultation and Therapy, 6, 373-394.

Wilson, K. G., Sandoz, E. K., Kitchens, J., \& Roberts, M. (2010). The Valued Living Questionnaire: Defining and measuring valued action within a behavioral framework. The Psychological Record, 60, 249-272. 
